\title{
Naissance et déclin d'une élite locale : la Banque des Cantons de l'Est, 1859-1912
}

\section{Ronald Rudin}

Volume 38, numéro 2, automne 1984

Bourgeoisies et Petites Bourgeoisies

URI : https://id.erudit.org/iderudit/304258ar

DOI : https://doi.org/10.7202/304258ar

Aller au sommaire du numéro

Éditeur(s)

Institut d'histoire de l'Amérique française

ISSN

0035-2357 (imprimé)

1492-1383 (numérique)

Découvrir la revue

Citer cet article

Rudin, R. (1984). Naissance et déclin d'une élite locale : la Banque des Cantons de l'Est, 1859-1912. Revue d'histoire de l'Amérique française, 38(2), 165-179.

https://doi.org/10.7202/304258ar d'utilisation que vous pouvez consulter en ligne.

https://apropos.erudit.org/fr/usagers/politique-dutilisation/ 


\title{
NAISSANCE ET DÉCLIN D'UNE ÉLITE LOCALE: LA BANQUE DES CANTONS DE L'EST, 1859-1912
}

\author{
RONALD RUDIN \\ Département d'histoire \\ Université Concordia
}

Avant la Première Guerre mondiale, le système bancaire canadien regroupait divers types d'établissements: banques jouissant d'un actif considérable et banques disposant de ressources plus modestes, banques faisant leurs affaires principalement en anglais et d'autres en français, banques desservant les grands centres urbains et d'autres, de petites villes. En 1914, toutefois, il n'y avait plus lieu de retenir cette dernière opposition. Antérieurement, en 1881, par exemple, certaines banques avaient leur siège social dans des villes aussi peu importantes que Pictou (Nouvelle-Ecosse) et Bowmanville (Ontario). Au Québec, trois banques avaient établi leur siège social à St-Jean, à St-Hyacinthe et à Sherbrooke. On pouvait dénombrer au total dix banques dont le siège social était situé dans des villes d'importance vraiment secondaire; or, les actifs totaux de ces établissements se chiffraient à 14 millions \$. À la fin de 1912, toutefois, une seule banque à charte canadienne, la Weyburn Security Bank de la Saskatchewan, était implantée en dehors d'un grand centre avec un actif inférieur à 2 millions $\$^{1}$. Les banques des petites villes subissaient les contrecoups des baisses répétées de l'activité économique, de la mauvaise gestion d'administrateurs qui voulaient parfois traiter un gros volume d'affaires avec un capital restreint et, surtout, des désirs d'expansion des grandes banques.

La Banque des Cantons de l'Est, implantée à Sherbrooke, représente l'un des établissements qui, parmi les banques de petites villes, a remporté le plus grand succès. Fondée en 1859 , elle a poursuivi ses activités jusqu'en 1912, époque à laquelle elle exploitait 103 succursales et disposait d'un actif de plus de 25 millions \$. Aucune autre banque de petite ville n'avait su se hisser à un niveau comparable.

L'acquisition en 1912 de la Banque des Cantons de l'Est par la Banque de commerce canadienne a d'ailleurs constitué la plus importante fusion jusque-là dans le secteur bancaire. L'importance de cette

\footnotetext{
1 Dans cet article, toutes les données au sujet des actifs et des passifs des banques sont tirées des rapports mensuels publiés dans la Gazette du Canada. À la fin de 1912, on trouvait des sièges sociaux de banque à Montréal, à St-Jean (Nouveau-Brunswick), à Toronto, à Québec, à Hamilton, à Ottawa, à Winnipeg et à Vancouver; seule la Weyburn Security Bank avait son siège social dans une petite ville, soit à Weyburn, en Saskatchewan.
} 
banque ne tenait pas uniquement à sa longue existence, passablement fructueuse. Fondée par les hommes d'affaires les plus influents de la région, la Banque des Cantons de l'Est a été, à l'origine, financée par les résidents des environs. Au fil des ans, toutefois, la diminution de la population anglophone de la région, tant en termes relatifs qu'absolus, a entraîné la nécessité de trouver des capitaux à l'extérieur si bien que la mainmise de l'élite locale sur l'établissement s'est amoindrie. Ayant perdu ses assises régionales, la banque est devenue une proie facile pour une banque plus puissante désireuse d'en faire l'acquisition.

L'élite anglophone des Cantons de l'Est a vu son influence péricliter à la suite de la montée du capitalisme de monopole, mais son pouvoir s'est aussi affaibli en raison de l'apparition d'une majorité francophone dans la région. L'histoire de la Banque des Cantons de l'Est met bien en relief l'existence d'une élite dont le pouvoir s'est érodé progressivement pendant les années qui ont précédé la Première Guerre mondiale.

$$
* *
$$

Avec l'établissement d'une colonie à majorité francophone dans le Bas-Canada en 1791, les Cantons de l'Est se sont vus conférer une position privilégiée au vu de la politique coloniale de la Couronne britannique, désireuse de faire échec à la majorité francophone. Cette vaste région était exclue de la tenure seigneuriale dans l'Acte constitutionnel de 1791 de telle sorte que les colons anglophones, américains et britanniques, puissent s'y établir. Les efforts déployés pour inciter la venue de colons anglais dans la région ont reçu une impulsion nouvelle grâce à la cession, en 1833, d'une importante partie de ce territoire à la British American Land Company, pour une bouchée de pain. En dépit de ces mesures incitatives, cette vaste région était encore relativement peu peuplée au début des années 1850 : sa population totale s'élevait en effet à moins de 100000 habitants et Sherbrooke, la principale ville, comptait environ 3000 âmes. Si la population était clairsemée, elle demeurait néanmoins majoritairement anglophone, soit à $60 \%$ pour l'ensemble de la région et à $75 \%$ à Sherbrooke ${ }^{2}$.

Ce sont les chefs d'entreprise de la communauté anglophone de la région, en particulier ceux qui étaient associés à la société d'exploitation foncière, qui se sont mis à élaborer des plans visant à accélérer le développement des Cantons de l'Est. Leur rêve le plus cher était la construction d'un chemin de fer qui faciliterait le transport de voyageurs

\footnotetext{
2 Recensement du Canada, 1851. Les divisions du recensement de 1851 étaient quelque peu différentes de celles de la période 1861-1911, ce qui rend les comparaisons passablement difficiles. Après 1861, la région regroupait les comtés suivants: Brome, Compton, Drummond, Mégantic, Richmond, Shefford, Sherbrooke, Stanstead et Wolfe.
} 
et des marchandises en provenance et à destination de la région. Alexander Galt, commissaire de la Land Company dans les années 1840 et au début des années 1850 , s'est fait l'ardent défenseur de cette cause. Grâce à son influence et à celle d'autres hommes d'affaires de la région, le réseau du Chemin de fer du Saint-Laurent et de l'Atlantique a été construit au coeur même des Cantons de l'Est et traversait Sherbrooke au début des années 1850 .

Plusieurs de ces mêmes notables ont aussi cherché à doter la région des services financiers requis pour assurer son essor économique. Dès 1836, on a tenté d'obtenir, mais sans succès, l'ouverture d'une succursale de la Banque de Montréal à Sherbrooke ${ }^{3}$. En 1851, la City Bank, implantée à Montréal, avait une succursale à Sherbrooke, mais le sentiment général était que cet établissement saignait la région de ses fonds plutôt que de venir en aide aux entreprises locales. Finalement, en 1854, un groupe d'hommes d'affaires de la région ont adressé une requête au gouvernement provincial afin de se voir accorder une charte pour la fondation de la Banque des Cantons de l'Est. "Your petitioners, soulignaient-ils, suffer from the want of a local bank through which to transact their business. The commercial, mechanical and agricultural interests of the Townships are retarded and enterprise checked by the deprivation of accommodation. The managing class of the Montreal banks have little or no interest in our part of the province and often afford facilities for using money to their Montreal customers while the same privilege is not extended to country customers equally responsible.» ${ }^{4}$

Grâce à l'influence d'anciens promoteurs du Chemin de fer du Saint-Laurent et de l'Atlantique aussi connus que Alexander Galt et Benjamin Pomroy, homme d'affaires de la région, la Banque des Cantons de l'Est recevait sa charte en 1855. Le capital était fixé à 1,25 million \$, duquel 125000 \$ devaient être versés avant l'inauguration de la banque; le solde, quant à lui, devait être réglé dans les cinq premières années d'existence de l'établissement ${ }^{5}$. Dès le début, les instigateurs du projet ont cherché des capitaux auprès d'investisseurs disposant de ressources assez modestes, en particulier chez les fermiers des environs. Afin de les encourager à investir auprès d'une banque dirigée par des hommes d'affaires prospères, la charte précisait que les droits de vote des actionnaires devaient être pondérés de manière à limiter la proportion des voix des grands investisseurs. C'est ainsi qu'aucun actionnaire n'avait droit à plus de vingt voix dans les réunions et ce, en dépit du nombre d'actions qu'il détenait effectivement ${ }^{6}$.

\footnotetext{
3 Musée McCord, Hale Papers, lettre d'Edward Hale à P. McGill, 22 février 1836.

4 Archives publiques du Canada (APC), correspondance du secrétaire de la province, vol. CCCLVIII, 15 octobre 1854.

5 Statuts du Canada, 1855. c. 206.

6 Ibid.. Ces clauses, qui ont eu une influence sur la répartition des voix entre les actionnaires, ont été annulées par la Loi sur les banques de 1871, qui accordait une voix par action.
} 
Pomroy a parcouru la région, de ville en ville, afin de trouver preneur pour les actions de la banque, mais il n'obtint pas beaucoup de succès ${ }^{7}$. En 1858, un amendement apporté à la charte a permis de ramener à $100000 \$$ le capital initial requis et à $400000 \$$ le capital total à verser dans un délai de cinq ans ${ }^{8}$. La banque n'a pu toutefois ouvrir ses portes qu'en 1859 et ce, seulement en raison du fait, discutable d'ailleurs, que les débentures municipales de Sherbrooke ont été acceptées en paiement d'actions de la banque.

En 1856, la ville de Sherbrooke devint titulaire de $80000 \$$ de débentures grâce au Fonds d'emprunt municipal. Ce fonds avait été institué en 1852 afin de permettre aux municipalités du Haut-Canada d'emprunter de la province; il fut étendu au Bas-Canada en 1854. Les municipalités, qui devaient normalement payer $8 \%$ d'intérêt sur leurs obligations, pouvaient recevoir de la province des débentures sur lesquelles elles n'avaient à verser que $6 \%$. Pour obtenir ces débentures, elles devaient présenter une demande à la province en précisant les fins auxquelles elles étaient destinées. Elles pouvaient disposer de ces obligations à leur gré à la condition de les utiliser pour les fins énoncées dans la formule de demande, d'effectuer régulièrement des versements à la province pour payer l'intérêt et de constituer un fonds d'amortissement ${ }^{9}$.

Dans le cas de Sherbrooke, la formule de demande ne comportait aucune mention de l'appui qu'elle entendait accorder à une banque. Quoi qu'il en soit, au début de 1859 , comme la banque avait peine à réunir le capital nécessaire, les débentures de Sherbrooke ont été offertes au grand public afin de permettre aux résidents de les utiliser en paiement d'actions de la Banque des Cantons de l'Est. La banque savait que ces obligations se négociaient à l'époque à une valeur inférieure à leur valeur nominale, mais comme on tenait à ce que la banque ouvre enfin ses portes, elles furent acceptées, comme l'atteste le procès-verbal de la réunion des administrateurs tenue le 20 juin 1859, «at their per value without reference to their current value in the market» ${ }^{10}$. Edward D. Worthington, par exemple, qui était titulaire de débentures de $200 £$, s'en servit pour donner un acompte de $10 \%$ sur ses actions bien que la valeur au prix du marché des débentures était inférieure à $200 £$. "The law provided that owners of municipal loan debentures might pay for their bank stock in this security», faisait remarquer Victor Ross, historien de la Banque de commerce canadienne, mais sans aucu-

\footnotetext{
7 Victor Ross, History of the Canadian Bank of Commerce (Toronto 1920), I: 363.

8 Statuts du Canada, 1858 , c. 22.

9 Pour plus de détails sur le Fonds d'emprunt municipal, voir Albert Faucher, Histoire économique et unité canadienne (Montréal, 1970), 83-106.

${ }_{10}$ Archives de la Banque de commerce canadienne impériale (BCCI), Banque des Cantons de l'Est (BCE), procès-verbal du Conseil d'administration, 20 juin 1859. L'auteur tient à remercier ici le service des archives de la BCCI de lui avoir permis de consulter les documents de la Banque des Cantons de l'Est. Sans cette permission, le présent article n'aurait pas pu voir le jour.
} 
nement faire mention de la loi qui légitimait un tel mode de paiement ${ }^{11}$. Il n'y avait certainement rien qui permît l'utilisation d'une valeur mobilière dont le cours était insuffisant pour satisfaire aux exigences énoncées dans la charte de la banque.

Les irrégularités, pour ne pas dire les illégalités, qui ont été commises par l'utilisation des débentures de Sherbrooke, ont fait tiquer plus d'un membre de la collectivité locale. Pour la Sherbrooke Gazette, par exemple, les débentures avaient été acquises «under a false pretense» et «diverted to a purpose foreign to the expressed intention of the Legislature» ${ }^{12}$. Dans une lettre au rédacteur en chef de la Gazette, un lecteur faisait remarquer que le recours à ces obligations indiquait «either a lack of capital or a lack of faith that prevent[ed] the subscription of a sufficient amount of stock to put the bank in operation» ${ }^{13}$. Alexander Galt, alors ministre des Finances, à qui incombait la responsabilité de veiller à ce que les obligations servent aux fins auxquelles elles étaient destinées, s'est plaint à son tour de l'usage qu'on réservait aux débentures. Voici en quels termes il s'exprime dans une lettre adressée à Pomroy au début de 1859: «I am willing to be responsible for $\$ 10000$ in the bank provided that other bona fide subscriptions be obtained to put the bank in operation. But it is quite impossible for me in my present position [as Minister of Finance] to give any sanction by [my] subscription of stock to the use that is proposed to be made to the municipal debentures. If they are to form part of the capital of the bank..., I must decline taking any stock.» ${ }^{14}$ En dépit de ses réticences, Galt a souscrit $10000 \$$ d'actions et la Banque des Cantons de l'Est a ouvert ses portes en septembre 1859.

À l'inauguration de la banque, environ $90 \%$ du capital était détenu par des Estriens, qui ont à leur tour choisi comme administrateurs certains des hommes d'affaires les plus influents de la région. En tête de liste figurait Benjamin Pomroy de Compton, qui fut nommé président de la banque. Dans les années 1860, Pomroy a aussi contribué à la création de la fabrique de lainages Paton à Sherbrooke et à la promotion du Chemin de fer de la Vallée de la Massawippi, qui devait assurer la liaison entre Lennoxville et la frontière américaine. Pour le projet Paton, Pomroy a travaillé en collaboration avec deux autres des membres de la première équipe de direction de la Banque, J.H. Pope et R.W. Heneker; pour le projet Massawippi, il reçut la collaboration d'un autre administrateur de la banque, A.A. Adams. Heneker, qui avait

\footnotetext{
11 Ross, I: $362-3$. Tout porte à croire que Ross fait ici allusion à la clause de la charte de la banque qui lui imposait d'investir $10 \%$ de son capital dans des débentures comme celles du Fonds municipal d'emprunt. Il fait l'erreur de confondre l'emploi de ces débentures en guise de paiement d'actions de la banque et la manière dont la banque devait investir les fonds reçus en paiement de ses actions.

12 Sherbrooke Gazette, 15 janvier 1859.

13 Ibid., $1^{\text {er }}$ janvier 1859.

14 APC, Galt Papers, vol. 10, lettre de Galt à Pomroy, 10 février 1859.
} 
succédé à Galt au poste de commissaire de la British American Land Company, a aussi participé à la promotion du Chemin de fer international de Saint-François et Mégantic, projet auquel Pope a également été associé. Il s'agissait, on le voit, d'un groupe d'hommes d'affaires très unis, dont le rôle au sein de la Banque des Cantons de l'Est ne constituait qu'une facette de leurs multiples activités dans la région. Ils représentaient ce que Paul-André Linteau a appelé la «moyenne bourgeoisie» 15

Durant les premières années d'existence de la banque, ces hommes influents ont continué à se démener pour réunir le capital fixé dans la charte. Afin de mieux convaincre les fermiers que la banque pouvait servir leurs intérêts, on adopta peu après l'ouverture un règlement selon lequel l'endettement de toute personne ou société ne pouvait dépasser $10000 \$^{16}$. La volonté de la banque d'appliquer ce règlement à la lettre est clairement démontrée par l'avertissement qu'elle a envoyé à J.H. Pope en 1860, l'enjoignant de réduire son endettement de $13000 \$$ à $10000 \${ }^{17}$. Mais le capital mit du temps à être réuni et lorsqu'il devint évident que les $400000 \$$ ne pourraient être versés pour 1864, on fit adopter un autre amendement afin de proroger le délai jusqu'en $1867^{18}$.

Avec son capital restreint, la banque dut, semble-t-il, limiter ses activités tout au cours des années 1860 à de modestes prêts aux agriculteurs et commerçants de la région ${ }^{19}$. On avança des fonds aux exploitants des mines de cuivre de la région, qui connurent des années grasses pendant la Guerre civile américaine, mais ces sommes étaient négligeables si on les compare aux prêts consentis à l'industrie minière après la dépression des années $1870^{20}$. En 1869 , soit dix ans après son inauguration, la banque disposait encore d'un actif inférieur à $750000 \$$. La situation a toutefois changé au fur et à mesure que Sherbrooke s'est transformée, vers la fin des années 1860, en un centre industriel important avec l'ouverture de la fabrique de lainages Paton. Cette manufacture employait 200 personnes en 1871, soit à peu près autant d'employés que l'ensemble des travailleurs dans les autres usines de Sherbrooke en

\footnotetext{
15 Paul-André Linteau, «Quelques réflexions autour de la bourgeoisie québécoise, 18501914», Revue d' histoire de l'Amérique française, 30, 1 (juin 1976): 55-56.

16 BCCI, BCE, procès-verbal du Conseil d'administration, 19 septembre 1859.

17 Ibid., 5 octobre 1860

18 Statuts du Canada, 1864, c. 82.

19 Dans cet article, toutes les données au sujet des prêts consentis par la banque sont tirées des procès-verbaux des conseils d'administration. Les registres ne sont toutefois pas complets. Certains ne nous donnent aucun détail au sujet des prêts accordés. Nous ne disposons, par exemple, de renseignements que pour les années 1859-66, 1870, 1876 et 1908-12. Même si les prêts qui ont été discutés font l'objet d'une énumération, on ne peut supposer pour autant que tous les prêts consentis par la banque sont mentionnés dans les procès-verbaux. Dans les premières années, les activités de la banque étaient restreintes; les renseignements sont donc plus complets. En 1908, toutefois, seules les transactions les plus importantes devaient être discutées par les administrateurs.

20 E.C. Woodley, «The Story of the Eastern Townships Bank», Report of the Missisquoi County Historical Society, VI (1960): 58.
} 
1866. Grâce à l'activité économique suscitée par la fabrique Paton, la population sherbrookoise est passée de 3000 en 1861 à près de 4500 en 1871. Au début des années 1870 , les administrateurs de la banque exhibaient leur optimisme en affirmant que Sherbrooke allait devenir «the most important seat of manufactures in the Dominion outside Montreal» $»^{21}$

Ce volume d'affaires accru nécessitait des fonds supplémentaires et, entre 1871 et 1875 , le capital de la banque est passé de $400000 \$$ a 1 million \$. En augmentant ainsi son capital, la banque suivait la même voie que le milieu bancaire dans son ensemble au début des années 1870. Toutefois, ces fonds supplémentaires ont nettement eu pour effet d'affaiblir les assises régionales de la banque, moins de $80 \%$ des actions étant détenus par des Estriens en 1872. La majeure partie du capital d'appoint fut trouvée à Montréal ou dans les Etats avoisinants de la Nouvelle-Angleterre, fait guère surprenant si l'on songe aux revers essuyés par la banque au moment où elle a tenté de réunir le capital initial dans la région.

D'autres changements ont résulté de la venue, en 1874, de Heneker, alors président de la fabrique Paton, lequel venait prendre la succession de Pomroy au poste de président. Heneker imposa un style de gestion audacieux, dont témoigne sa proposition de 1876 portant sur l'achat de la Banque métropolitaine, implantée à Montréal. Les administrateurs de la banque jugèrent le projet imprudent, compte tenu de la crise économique qui sévissait alors; Heneker réussit toutefois à faire accepter aux administrateurs une modification des règlements de la banque qui eut pour effet de supprimer le plafond d'endettement ${ }^{22}$. Lorsque la crise se fut résorbée vers la fin des années 1870 , Heneker était donc en position de prêter des sommes importantes aux principales

TABLEAU I

RÉPARTITION DES ACTIONS DE LA BANQUE DES CANTONS DE L'EST, 1863-1912

$\begin{array}{ccccc}\text { Année } & \text { Capital versé } & \text { Cantons de l'Est } & \text { Montréal } & \text { États-Unis } \\ 1863 & 240250 \$ & 89 \% & 7 \% & 1 \% \\ 1872 & 567675 \$ & 79 \% & 12 \% & 3 \% \\ 1882 & 1397659 \$ & 63 \% & 12 \% & 9 \% \\ 1892 & 1487582 \$ & 57 \% & 10 \% & 18 \% \\ 1902 & 1955225 \$ & 53 \% & 7 \% & 28 \% \\ 1912 & 3000000 \$ & 44 \% & 6 \% & 38 \%\end{array}$

Sources, Documents de session, 1863-1912

$21 \quad$ BCCI, BCE, Annual report, 1871.

22 Ibid., procès-verbaux des réunions du Conseil d'administration, 18 juillet 1876; 5 juin 1875 . 
entreprises de Sherbrooke et de la région. Comme on pouvait s'y attendre, la fabrique Paton a été l'une des premières entreprises à bénéficier d'un prêt important négocié par Heneker, soit la somme de $275000 \$$ en $1880^{23}$. Vers le milieu des années 1890 , plusieurs autres avances ont été consenties à des entreprises locales. En 1895, par exemple, le total des emprunts contractés par le Chemin de fer du comté de Drummond et le Chemin de fer d'Hereford auprès de la Banque des Cantons de l'Est s'élevait à près de $500000 \$^{24}$. La banque entretenait en outre d'étroites relations avec les entreprises minières, les sociétés d'exploitation forestière et les usines de pâtes et papiers. Dans le secteur minier, elle comptait parmi ses clients plusieurs grands exploitants de mines d'amiante; une avance qui s'élevait à près de $100000 \$$ en 1899 avait en outre été consentie à la Jenckes Machine Company de Sherbrooke, un fabricant de matériel d'exploitation minière employé dans les mines de la région ${ }^{25}$. L'un des clients encore plus importants de la banque était la Royal Paper Mills, qui exploitait une grande usine non loin de Sherbrooke, à East Angus ${ }^{26}$

En 1895, l'actif de la Banque des Cantons de l'Est dépassait 6 millions \$ et son capital atteignait la limite autorisée de 1,5 million \$. En dépit de cette croissance, la banque demeurait un établissement à vocation régionale, ses dix succursales étant situées dans les Cantons de l'Est. Mais à partir de la fin des années 1890, la situation allait changer en raison de nombreux facteurs. Mentionnons d'abord la diminution progressive du rôle d'Estriens parmi les actionnaires: début des années 1890 , seulement $57 \%$ du capital étaient encore entre les mains d'Estriens alors que le nombre d'actionnaires de la Nouvelle-Angleterre allait en s'accroissant. Les liens étroits entre les Cantons de l'Est et les États américains au Sud étaient manifestes en 1859 dans la mesure où tous les membres de la première équipe de direction de la banque, à l'exception de Heneker, avaient des ancêtres en Nouvelle-Angleterre. Forts d'une participation accrue dans la banque, les résidents de la Nouvelle-Angleterre ont voulu se faire représenter au conseil d'administration et c'est ainsi qu'en 1894, John Foster de Derby Line (Vermont) est devenu le premier administrateur non estrien.

Peu après la nomination de Foster, la banque a ouvert, en 1895, sa première succursale en dehors de la région, soit à Saint-Hyacinthe. On pouvait s'attendre en effet à ce que des investisseurs de l'extérieur appuient cette expansion, surtout si l'on songe à la situation financière de la banque au début des années 1890 . Tout au cours des années 1860 ,

\footnotetext{
23 Ibid., 18 août 1880; Cleyn and Tinker Co. (Huntingdon, Québec), Paton Manufacturing Company, procès-verbal du Conseil d'administration, 18 août 1880 1895.

25 Ibid., 6 juin 1889

26 Ibid., 6 décembre 1904.
} 
1870 et 1880 , la banque réalisait généralement des bénéfices et distribuait des dividendes supérieurs à ceux des autres banques québécoises de même importance ${ }^{27}$. Elle s'était en outre constitué un fonds de réserve de plus de $500000 \$$ en 1890. Au début des années 1890, cependant, pour la première fois de son existence, elle affichait des bénéfices inférieurs à ceux des banques comparables et ce, pendant trois années consécutives, en raison vraisemblablement des embarras financiers de plusieurs de ses clients locaux importants. La fabrique Paton, par exemple, connaissait, comme les autres fabriques de lainages et filatures canadiennes de l'époque, un surcroît de production. L'entreprise a essuyé des pertes durant une bonne partie des années 1890, a temporairement fermé ses portes en 1894 et n'a réussi à réaliser des bénéfices comparables à ceux des années 1880 qu'avec le début de la Première Guerre mondiale ${ }^{28}$. Les affaires de l'usine de papier d'East Angus étaient, elles aussi, chancelantes. En 1891, le fondateur de l'usine, William Angus, a vendu son entreprise à la Royal Pulp and Paper Company, qui a dû à son tour procéder à une liquidation en 1893 au profit de la Royal Paper Mills ${ }^{29}$.

Au début des années 1890, les administrateurs locaux de la banque voyaient tout aussi bien que les investisseurs de l'extérieur les bénéfices baisser. Heneker devait reconnaître qu'un grand nombre de ses investissements avaient mal tourné; le problème qui se posait était de trouver des fonds pour financer de nouveaux investissements. En 1890, les dépôts d'épargne occupaient une place de plus en plus importante dans le passif des banques canadiennes. C'étaient donc ces dépôts d'épargne, et non une augmentation du capital, qui allaient permettre de nouveaux investissements; mais en 1895, les dépôts d'épargne ne représentaient que $40 \%$ de tout le passif de la Banque des Cantons de l'Est et non $48 \%$ comme dans les banques québécoises de taille comparable. Tout au cours de ses premières années d'existence, elle avait tablé sur le monopole qu'elle exerçait en matière de services bancaires dans la région pour attirer chez elle les épargnes des Estriens. Au début du siècle, cependant, moins de la moitié de l'ensemble des succursales bancaires de la région appartenaient à la Banque des Cantons de l'Est (voir tableau III). De plus, la population anglophone de la région, clien-

\footnotetext{
27 Il s'agit, partout dans cet article, des bénéfices nets exprimés en pourcentage du capital versé. C'était la manière d'analyser la rentabilité d'une banque durant la période étudiée.

Pour trouver des «banques comparables», nous avons réparti, par année, toutes les banques implantées au Québec en six catégories selon leur actif total: moins de 1 million $\$$, de 1 à 5 millions $\$$, de 5 à 10 millions $\$$, de 10 à 25 millions $\$$, de 25 à 100 millions $\$$ et plus de 100 millions \$. Le groupe dans lequel a été placée la Banque des Cantons de l'Est a été choisi en fonction de l'actif total de la banque pour l'année envisagée. Ce système de comparaison nous a permis d'étudier la rentabilité de la banque, ses dividendes ainsi que la nature de ses actifs et de ses passifs.

28 Cleyn and Tinker, Paton Manufacturing Company, Financial reports, 1869-1914; Prix courant, 24 mai 1894.

29 L.S. Channell, History of Compton County (Cookshire, 1896), 230.
} 
tèle habituelle de la banque, diminuait en importance tant en termes relatifs qu'absolus ${ }^{30}$. En 1871, la région regroupait plus de 60000 non francophones, qui représentaient $52 \%$ de la population; en 1901, ce chiffre n'atteignait même plus les 60000 , les francophones représentant désormais la majorité.

Il devint évident que si la banque voulait se renflouer, il lui fallait trouver des épargnes à l'extérieur de la région. L'ouverture de la succursale de Saint-Hyacinthe en 1895 a constitué un premier pas dans cette direction. L'initiative était d'autant plus frappante que les francophones constituaient une écrasante majorité dans cette ville. Or, tout au long de son existence, la banque avait toujours eu des actionnaires et des administrateurs anglophones et jusqu'en 1895, jamais une succursale n'avait été ouverte dans une ville dont plus de $75 \%$ des résidents étaient francophones ${ }^{31}$. Au milieu des années 1890, toutefois, la banque ne pouvait plus faire la difficile en face d'une bonne occasion d'affaires. Or c'est justement ce qui s'est produit lorsque la Banque du Peuple a fermé, en raison de la suspension de ses activités, sa succursale de Saint-Hyacinthe, laissant ainsi un vide à combler.

En 1901, six des seize succursales de la banque se trouvaient en dehors des Cantons de l'Est, dont l'une à Montréal et deux en Colombie britannique. L'ouverture de la succursale montréalaise comme celle des succursales de la Colombie britannique avaient été amenées par une nouvelle détérioration des perspectives d'affaires dans les Cantons de l'Est. C'est ce qu'expliquait à l'époque le directeur général adjoint de la banque en parlant de la succursale montréalaise: «The process of

TABLEAU II

POPULATION NON FRANCOPHONE DES CANTONS DE L'EST, 1861-1911

Population non francophone

1861

1871

1881

1891

1911
61325

61962

63830

58918

59947
$\%$ de la population totale de la région

$56 \%$

$52 \%$

$40 \%$

$31 \%$

$28 \%$

Source: Recensement du Canada, 1871-1911

\footnotetext{
30 Sur la question de la diminution du nombre d'anglophones dans la région, voir J.I. Little, «Watching the Frontier Disappear: English-Speaking Reaction to French-Canadian Colonization in the Eastern Townships, 1844-1890», Revue d'études canadiennes, XV (1880-81): 93-111.

31 Les données sur les succursales de la banque et les caractéristiques démographiques des villes où elles étaient installées proviennent de deux sources: les répertoires de succursales bancaires publiés tout au cours de cette période et Recensement du Canada.
} 
centralization of large business interests in (Montreal) has been going on for some years and gradually the business which the bank has nurtured and supported in its territory (has) been transfered to Montreal, and not having an office there we have lost the business we should properly have had.» ${ }^{32}$ L'ouverture de la succursale montréalaise était donc un signe de l'érosion du pouvoir économique de l'élite estrienne dans la région même. L'ouverture des succursales en Colombie britannique témoignait, par contre, de l'expansion de plusieurs entreprises locales sur le marché de l'Ouest. En 1899, par exemple, les administrateurs de la banque recevaient une lettre d'un actionnaire de longue date, S.C.H. Miner de Granby, les informant qu'il était sur le point d'établir une fonderie à Grand Forks, en Colombie britannique. Il priait la banque d'y ouvrir une succursale afin d'être, pour reprendre ses mots, «the first in the field» et assurait les administrateurs qu'elle se verrait confier «the business of the smelting company and the mines with which he was connected» ${ }^{33}$. Cet exode des fonds et des compétences des Cantons de l'Est vers les régions minières de la Colombie britannique se trouvait renforcé par l'ouverture d'une filiale de la Jenckes Machine Company dans l'Ouest. La banque pouvait difficilement se permettre de laisser passer pareilles occasions.

Ces diverses tendances dans la conduite des affaires de la banque se sont accentuées au cours de ses dix dernières années d'existence. Au fur et à mesure qu'augmentait son capital pour bientôt atteindre 3 millions \$, la participation des résidents de la Nouvelle-Angleterre devenait plus importante, au détriment de celle des Estriens. En 1912, seulement $44 \%$ du capital de la banque étaient encore entre les mains d'Estriens. Signe de cette éclipse progressive des investisseurs locaux, cinq des onze administrateurs en poste en 1912 venaient de l'extérieur. Ces hommes n'ont d'ailleurs pas essayé de stopper l'élargissement des activités de la banque hors de sa sphère traditionnelle, le milieu anglophone des Cantons de l'Est, du reste en régression continuelle. Entre 1902 et 1911, sous la direction du successeur de Heneker, William Farwell, le nombre de succursales est passé à 89, la majorité des nouvelles agences ayant été ouvertes à l'extérieur des Cantons de l'Est. Fidèles à leurs origines, toutefois, les succursales du Manitoba, de l'Alberta et de la Colombie britannique ont accordé des prêts substantiels aux mêmes secteurs de l'économie à qui la banque avait prêté main forte dans les Cantons de l'Est, à savoir les secteurs de l'exploitation minière et forestière $^{34}$. En outre, les succursales de l'Ouest ont cherché à obtenir

32 BCCI, BCE, Annual Report, 1901.

33 Ibid., procès-verbal du Conseil d'administration, 5 juin 1899.

34 Au cours de ses réunions en 1909, par exemple, le Conseil d'administration de la banque a consenti des prêts de plus de $100000 \$$ aux entreprises suivantes: la Bell Asbestos Company (Thetford-Mines), la Brampton Pulp and Paper Company (Sherbrooke), l'International Coal and Coke Company (Coleman, C. b.) et la Great West Lumber Company (Winnipeg). Les villes entre parenthèses indiquent quelle succursale a consenti le prêt. 
la clientèle des Estriens anglophones devenus agriculteurs dans les provinces des Prairies ${ }^{35}$. Au Québec, la banque a ouvert de plus en plus de succursales dans les villes où les francophones représentaient une écrasante majorité, reconnaissant ainsi la stagnation de la population anglophone.

En 1911, la Banque des Cantons de l'Est était devenue un établissement national et non plus seulement régional. La politique d'expansion en vue de susciter des dépôts d'épargne, qui avait été instaurée vers le milieu des années 1890, avait remporté un tel succès que ces dépôts représentaient, en 1911, 54\% de tout le passif de la banque. Seule une autre banque québécoise affichait un pourcentage plus élevé. D'autres faits témoignaient du succès de la banque dans la conduite de ses affaires: au cours de la première décennie du $\mathrm{XX}^{\mathrm{e}}$ siècle, les dividendes versés n'ont jamais été inférieurs à $8 \%$, les bénéfices nets ont toujours dépassé $12 \%$ du capital versé et le fonds de réserve a augmenté progressivement pour atteindre 2,4 millions \$ en 1911. Les dividendes étaient généralement supérieurs à ceux des autres banques québécoises de même importance, mais les bénéfices réalisés, aussi intéressants qu'ils aient pu sembler à première vue, étaient inférieurs à ceux des banques comparables et ce, durant huit des neuf dernières années d'existence de la banque. Non pas que les affaires de la banque ne fussent pas rentables, mais elles l'étaient moins que celles des établissements ayant leur siège social dans l'un des grands centres urbains du Canada et bénéficiant d'un accès facile aux renseignements requis et aux clients. Afin de résoudre ce problème, les administrateurs songèrent sérieusement, en 1911, à déménager le siège social de la banque à Montréal $^{36}$.

\section{TABLEAU III}

SUCCURSALES DE LA BANQUE DES CANTONS DE L'EST, 1871-1911

\begin{tabular}{cccc} 
\% des succursales de la & \% de toutes les \\
Année & $\begin{array}{c}\text { Banque des Cantons de l'Est } \\
\text { dans les villes du Québec } \\
\text { drancophones à } 75 \%\end{array}$ & $\begin{array}{c}\text { des Cantons } \\
\text { de l'Est }\end{array}$ & $\begin{array}{c}\text { succursales estriennes } \\
\text { exploitées par la } \\
\text { Banque des Cantons } \\
\text { de l'Est }\end{array}$ \\
1871 & $0 \%$ & $100 \%$ & $80 \%$ \\
1881 & $0 \%$ & $100 \%$ & $78 \%$ \\
1891 & $*$ & $100 \%$ & $62 \%$ \\
1901 & $7 \%$ & $63 \%$ & $40 \%$ \\
1911 & $47 \%$ & $56 \%$ & $62 \%$ \\
* Données non disponibles & & \\
\hline
\end{tabular}

35 Woodley, 59-60.

36 BCCI, BCE, procès-verbal du Conseil d'administration, 29 mai 1911. 
Le projet n'a jamais vu le jour en raison de l'acquisition de la banque par la Banque de commerce en 1912. L'offre d'achat a été présentée vers la fin de 1911, au retour d'un voyage dans l'Ouest d'un groupe d'administrateurs, désormais persuadés que seule l'ouverture de nombreuses autres succursales dans cette partie du pays allait permettre à la banque de conserver sa part du marché en face de la vive concurrence faite par les grandes banques. Ce projet d'expansion allait nécessiter de nouveaux capitaux et les administrateurs, voyant que les bénéfices nets de la banque exprimés en pourcentage du capital versé étaient en deçà des bénéfices des autres banques de taille comparable, devaient s'interroger sur sa rentabilité. Les plus hésitants étaient les administrateurs qui résidaient à l'extérieur de la région et représentaient, en 1911, la majorité des actionnaires. Ces personnes n'avaient aucune attache sentimentale à la Banque des Cantons de l'Est et ne tenaient pas à la garder ouverte à tout prix. La première offre d'achat, effectuée par la Banque de Montréal en 1905, avait été rejetée parce que les bénéfices réalisés étaient encore acceptables, mais, après les années de bénéfices maigres, les administrateurs de l'extérieur se sont faits les ardents défenseurs de la proposition de la Banque de Commerce ${ }^{37}$. En fait, les deux seuls administrateurs qui se sont opposés à la vente étaient des Estriens ${ }^{38}$.

Si la seule opposition à la fusion est venue d'administrateurs locaux, la majorité des administrateurs provenant de la région ont reconnu que l'offre faite par la Banque de Commerce était valable et c'est pourquoi ils se sont empressés de l'accepter. En gros, la Banque de Commerce offrait d'échanger 3 millions $\$$ de ses propres actions contre 3 millions \$ d'actions de la Banque des Cantons de l'Est. La proposition était intéressante car les actions de la Banque de Commerce se traitaient à un cours supérieur à celui des actions de la Banque des Cantons de l'Est et rapportaient des dividendes plus élevés. Et pour mieux dorer la pilule, la Banque de Commerce acceptait en plus de faire siéger trois ou quatre des administrateurs de la Banque des Cantons de l'Est à son propre conseil d'administration et de désigner les autres administrateurs comme membres d'un comité consultatif siégeant à Sherbrooke ${ }^{39}$.

La Banque de Commerce était disposée à faire une offre aussi alléchante parce qu'elle voulait se doter d'un réseau de succursales au Québec. Les succursales de l'Ouest offraient peu d'intérêt dans la mesure où elles faisaient souvent pour elle double emploi, ce qui n'était absolument pas le cas au Québec. En 1911, la Banque de Commerce ne comptait, en effet, que deux succursales dans la province, l'une à Mont-

37 Ibid., 7 juin 1905.

38 Ibid., 5 décembre 1911. bre 1911 .

Ibid., circulaire adressée aux actionnaires de la Banque des Cantons de l'Est, 21 décem- 
réal et l'autre à Québec; les succursales québécoises de la Banque des Cantons de l'Est allaient changer le tableau et permettre à la Banque de Commerce d'entrer en contact avec une clientèle francophone. D'après le fondateur du Mouvement des Caisses populaires, Alphonse Desjardins, le principal motif de la Banque de Commerce était, en effet, d'obtenir «la clientèle française... créée (par) la Eastern Town whips Bank» ${ }^{40}$. Cette dernière avait à ce point dévié de sa vocation régionale au service d'une clientèle anglophone qu'en 1911, elle s'est vu offrir une proposition généreuse en raison de sa clientèle francophone, proposition qu'elle a acceptée en raison de ses difficultés à conserver son marché dans l'Ouest.

$$
* *
$$

L'histoire de la Banque des Cantons de l'Est reflète les hauts et les bas de la population anglophone - en particulier de la «moyenne bourgeoisie» - de la région. La banque a été mise sur pied par des hommes d'affaires locaux qui ont cherché des capitaux auprès des résidents de la région, tâche qu'ils ont eu beaucoup de mal à remplir au début. Au fur et à mesure que la banque a pris de l'expansion, la majorité des fonds provenaient de l'extérieur. Alors que le financement des activités de la banque a nécessité, au début des années 1890, l'apport de fonds supplémentaires sous la forme de dépôts d'épargne, on ne pouvait plus compter sur la population anglophone des Cantons de l'Est, dégarnie par l'exode vers l'Ouest d'une partie de ses effectifs. Les personnes de l'extérieur qui ont siégé au conseil d'administration de la banque durant ses quinze dernières années d'existence prônaient la mise en oeuvre d'une politique d'expansion et les administrateurs provenant de la région pouvaient difficilement s'y opposer, étant données les conditions du marché. À la fin, la situation ne permettait plus aucun espoir: entraînée dans une concurrence inégale contre les grandes banques du Canada, la Banque des Cantons de l'Est a bel et bien disparu de la scène en 1912.

La baisse du pouvoir économique de l'élite locale, dont l'histoire de la banque n'est que le reflet, n'a pas été un phénomène isolé: dans tout le Canada, des banquiers de petites villes ont connu la même expérience que les administrateurs de la Banque des Cantons de l'Est. Pour les hommes d'affaires estriens, cette perte de contrôle sur la banque n'a été qu'un signe parmi d'autres de l'érosion générale de leur influence sur l'activité économique de la région. Heneker, par exemple, était à la fois président de la fabrique de lainages Paton et co-fondateur du Chemin de fer international de Saint-François et Mégantic, deux entreprises créées par des industriels locaux. Au début du siècle, toutefois, ces

40 Archives de la Fédération des Caisses populaires (Lévis), 22: 4d-1, Desjardins à Jules Dorion, 23 janvier 1913. 
deux sociétés étaient passées entre les mains d'influents hommes d'affaires montréalais ${ }^{41}$.

Le processus de consolidation de l'économie canadienne a contribué à saper l'influence de l'élite anglophone des Cantons de l'Est, mais la diminution de la population anglophone dans la région a également constitué un facteur déterminant. L'histoire de la Banque des Cantons de l'Est confirme à maints égards la relation entre la francisation de l'Estrie et le déclin de l'élite anglophone. La constitution progressive d'une majorité francophone a, elle aussi, sapé la mainmise de l'élite anglophone sur le pouvoir politique. Concrètement, ce changement démographique signifiait que l'élite anglophone n'était plus en mesure de monopoliser le poste de maire de la ville de Sherbrooke; c'est ainsi que dans les années 1880, la ville a élu son premier maire francophone. Alarmés par la situation, les hommes d'affaires locaux ont appuyé les sociétés de colonisation, susceptibles d'inciter des anglophones à venir s'installer dans la région. Heneker était justement à la tête du plus important de ces organismes, la Eastern Townships Colonization Society, fondée en 1882. Comme le soulignait l'un des conférenciers lors d'une réunion de la société: «Why should we allow ourselves to be crowded out by the French population which would assuredly be done if we fold(ed) our arms and (made) no effort to help ourselves.» ${ }^{42}$

En définitive, les hommes d'affaires anglophones des Cantons de l'Est n'ont pu ni retenir les anglophones dans la région, ni empêcher l'arrivée des francophones, ni conserver, à une époque de capitalisme de monopole, leur mainmise sur l'économie locale. L'histoire de la Banque des Cantons de l'Est nous permet de mieux saisir le déclin des élites locales dans l'ensemble du Canada ainsi que les circonstances particulières qui ont présidé à l'accélération de ce phénomène pour une certaine élite anglophone au Québec.

\footnotetext{
41 Pour un exposé plus complet sur le déclin de cette élite, voir R. Rudin, «The Transformation of the Eastern Townships of Richard William Heneker, 1855-1902», à paraitre dans la Revue d'études canadiennes.

42 Eastern Townships Historical Society, Eastern Townships Colonization Society, procèsverbaux, s.d.
} 